\title{
Caracterización psicosocial de cuidadores informales de adultos mayores con demencia
}

\author{
Psychosocial characterization of informal caregivers of older \\ adults suffering from dementia
}

\author{
Ana Margarita Espín Andrade \\ Máster en Psicología de la Salud. Asistente. Escuela Latinoamericana de Medicina. \\ La Habana, Cuba.
}

\begin{abstract}
RESUMEN
I ntroducciónEl envejecimiento de la población es sin duda la principal característica demográfica de Cuba, en la actualidad y también perspectivamente, dada sus implicaciones económicas y sociales.

Objetivos Describir características demográficas, sociales, psicológicas y otras de 61 cuidadores informales de personas que padecen demencia y que habían recibido atención en el Centro I beroamericano para la Tercera Edad en La Habana, de 2004 a 2005.

Métodos Se utilizó para la recogida de los datos el Cuestionario de Caracterización y la Escala Psicosocial del cuidador.

Resultados Los cuidadores informales estudiados se caracterizaron por ser en su mayoría del sexo femenino, entre los 40 y 59 años, hijos de los enfermos, casados, sin vínculo laboral en un alto porcentaje y predominio de universitarios en la muestra. La mayoría de los cuidadores atendían al enfermo por razones afectivas, no tenían experiencia de cuidar a un enfermo crónico y llevaban menos de 1 año en esta labor, no tenían información acerca de la enfermedad, padecían de problemas nerviosos, óseos y musculares, entre otros y su estrategia de afrontamiento era, funadamentalmente, la búsqueda de apoyo externo. La afectación de índole socioeconómica se encontró mayormente en el poco tiempo libre, problemas económicos y conflictos familiares. Los sentimientos negativos más frecuentes fueron la angustia o aflicción, la ira, el miedo y la desesperanza.

Conclusiones Los cuidadores informales tiene afectaciones múltiples relacionadas con la salud física y mental así como en el orden social y económico, por lo que se hace inminente la búsqueda de alternativas de apoyo puesto que, además de las dificultades planteadas, no cuentan con un mínimo de información acerca de la
\end{abstract}


demencia y de los problemas que tiene el adulto mayor al que ofrecen sus cuidados, a pesar de tener un nivel de enseñanza superior en su mayoría.

Palabras clave: Cuidador informal, demencia, anciano dependiente.

\section{ABSTRACTS}

Introduction Population aging is beyond any doubt the main demographic feature in Cuba at present day and in prospect, given its economic and social implications. Objectives To describe demographic, social, psychological and other kind of characteristics of 61 informal caregivers of persons suffering from dementia who had been treated at the Ibero-American Center of the Old Age located in the City of Havana from 2004 to 2005.

Methods The Characterization Questionnaire and the Psychosocial Scale of the Caregiver were the means to collect data.

Results The studied informal caregivers were females aged 40 to 59 years, daughters of the sick persons, married, non-working women at that time and predominantly with a university degree. The majority of caregivers took care of the sick people because of affectionate reasons, was inexperienced in managing a chronically ill patient and had spent less than a year in doing this task. They did not have any sort of information about the disease, suffered nervous, bone and muscle problems and their strategy to address the situation was mainly to look for thirdparty support. The socioeconomic effects basically centered in little leisure time, financial restrains and family conflicts. The most frequent negative feelings were anguish, anger, fear and hopelessness.

Conclusions Informal caregivers are affected in multiple ways related to their physical and mental health, as well as in the economic and social fields, so it is urgent to look for supporting alternatives since, in addition to the stated difficulties, the caregivers do not have basic information about dementia and the problems that the affected older person may face, despite the fact that most of them have higher educational level.

Key words: Informal caregiver, dementia, dependent aged person.

\section{NTRODUCCI ÓN}

El envejecimiento de la población es sin duda la principal característica demográfica de Cuba en la actualidad y también perspectivamente, dada sus implicaciones económicas y sociales. Poco más de 1,7 millones de personas tenían 60 años y más en el 2004, 15,4\% de la población. Este comportamiento debe intensificarse y para 2025, se pronostica que casi uno de cada cuatro cubanos será un adulto mayor, para ese entonces la edad promedio será de alrededor de 44 años y Cuba tendrá todas las características de un país envejecido. Ese es el efecto principal de la transición de la fecundidad y obviamente la sociedad tendrá que adecuar sus acciones, desde el punto de vista de su funcionamiento económico, social, cultural y otros. $^{1}$ 
Esta realidad impone grandes retos a toda la sociedad y en particular al sector de la salud ya que si bien se planifican acciones de promoción y prevención de enfermedades para la edad avanzada que tienen el objetivo de garantizar un envejecimiento satisfactorio, existe una porción de este grupo poblacional que presenta afecciones discapacitantes que dificultan el mantenimiento de una vida independiente y funcional.

Los avances científicos y tecnológicos han permitido el alargamiento de la vida y en muchos casos el disfrute de las personas ancianas de la última etapa de sus vidas. Pero así como la vejez no es un fenómeno homogéneo, la calidad de vida de los ancianos y su bienestar varían como resultado de esa diversidad. La edad es uno de los factores que condiciona la dependencia, influyendo de manera decisiva el aislamiento, la pobreza, la invalidez y el medio adverso.

Una de las afecciones que más limita las actividades de la vida diaria del adulto mayor es la demencia, esta es una enfermedad del sistema nervioso central que provoca pérdida de memoria y de otras funciones mentales superiores así como alteraciones de la conducta y la personalidad de los que la padecen.

El número de personas con demencia, a nivel mundial, crece rápidamente y las cifras lo demuestran porque en sólo 25 años, 34 millones de personas padecerán demencia. La cifra cobra significado cuando se coloca en el contexto del incremento de la expectativa de vida y el envejecimiento de la población en países en desarrollo. En el presente $66 \%$ ( 11 millones) de las personas con demencia vive en países en desarrollo y para el 2025 esta cifra se elevará hasta $75 \%$ ( 24 millones). ${ }^{2}$

Las demencias constituyen un serio problema de salud pública por ser una enfermedad de alto costo económico y social, por lo difícil y tardío de su diagnóstico y por su curso progresivo e invalidante. ${ }^{3}$

Esta enfermedad provoca alteraciones no sóloen el enfermo sino también en la familia ya que su aparición demanda de una redistribución de los roles familiares y genera un elevado estrés por las múltiples manifestaciones conductuales y el desconocimiento del proceso que las ocasiona, entre otros factores.

Una de las fuentes importantes de apoyo y cuidado en la vejez es la familia en la medida que es la sede de transferencias intergeneracionales de recursos materiales y de cuidados afectivos de suma importancia en la vida cotidiana de las personas mayores. La encuesta Salud, Bienestar y Envejecimiento (SABE) muestra una alta proporción de personas mayores que reciben apoyo familiar alcanzando la cifra de $93 \%$ en la Ciudad de La Habana. ${ }^{4}$

Dentro de la familia no todos asumen de igual manera el cuidado de sus enfermos. A los miembros que ocupan la máxima responsabilidad en el cuidado se les denomina "cuidadores". Se ha definido al cuidador como "aquella persona que asiste o cuida a otra afectada de cualquier tipo de discapacidad, minusvalía o incapacidad que le dificulta o impide el desarrollo normal de sus actividades vitales o de sus relaciones sociales." 5

Los cuidadores se afectan en el orden físico, psicológico y socioeconómico, lo que en conjunto conlleva a una "carga" que interfiere en el manejo adecuado del enfermo y en la propia evolución de la enfermedad, provocando al mismo tiempo el empeoramiento de la calidad de vida del cuidador.

La familia realiza el $80 \%$ del trabajo que requiere el cuidado del paciente con demencia y supone un importante "colchón" en la demanda de cuidados y servicios 
sanitarios pero en contrapartida sufre la importante carga emocional y de trabajo que supone este cuidado. ${ }^{6}$

Se ha definido la "carga" como el conjunto de problemas físicos, mentales y socioeconómicos que experimentan los cuidadores de enfermos crónicos que pueden afectar sus actividades de ocio, relaciones sociales, amistades, intimidad, equilibrio emocional y libertad. ${ }^{7}$

Se ha demostrado que son numerosas las variables que inciden en el aumento de la carga del cuidador, de un lado están las relacionadas con el enfermo, como son el grado de deterioro y/o pérdida de autonomía y la presencia de trastornos conductuales y psiquiátricos de difícil manejo, entre las fundamentales; de otro lado, las relacionadas con el propio cuidador entre las que se encuentran la edad, el sexo, el estilo de afrontamiento, la motivación para el cuidado, las redes de apoyo, el tiempo de cuidado y otros; y por último las derivadas de la relación afectiva cuidador-enfermo tanto previas como actuales.

Existen múltiples estudios que han arrojado un perfil del "cuidador principal" del paciente con demencia, también se han realizado mediciones de la carga y de otras alteraciones que con frecuencia sufren estos cuidadores. Los resultados de la mayoría de ellos coinciden en los diferentes países y regiones.

Así por ejemplo, se ha visto que por lo general son cuidadoras las hijas que se encuentran en la edad mediana de la vida, que tienen a su vez múltiples obligaciones sociales, laborales y familiares que le provocan alteraciones de diferentes tipos.

Los cuidadores informales de ancianos con demencia presentan altos niveles de depresión y ansiedad y en un elevado porcentaje de casos morbilidad psiquiátrica apreciable, aunque a niveles subclínicos. ${ }^{8}$

El presente trabajo tiene el objetivo de caracterizar a los cuidadores informales de los adultos mayores que sufren de demencia y describir la afectación socioeconómica y psicológica que presentan.

\section{MÉTODOS}

Se realizó un estudio descriptivo consistente en la caracterización de 61 cuidadores informales de adultos mayores con demencia (demencia vascular y enfermedad de Alzheimer), diagnosticados en el Centro I beroamericano para la Tercera Edad (CITED), de diciembre de 2004 a diciembre de 2005. Esta cifra correspondió a la totalidad de los sujetos que acudieron a la "consulta del cuidador" en ese período, remitidos por el servicio ambulatorio y la sala de geriatría del CITED y que cumplían con los siguientes requisitos:

- Ser cuidador informal de al menos un adulto mayor con demencia.

- Que la demencia haya sido diagnosticada en el servicio del CITED.

- Voluntad del cuidador de participar en el estudio.

Para dar salida a los objetivos del trabajo se realizó de manera individual en la consulta la aplicación de los siguientes instrumentos al cuidador: 
1. Cuestionario de Caracterización del Cuidador: es un instrumento de 15 ítems que recoge las variables del cuidador informal del adulto mayor con demencia: edad, sexo, estado civil, escolaridad, ocupación, número de personas a cuidar, motivación, información acerca de la enfermedad, estado de salud, experiencia como cuidador, estrategia de afrontamiento, tiempo de cuidador y parentesco. EI cuestionario fue validado desde el punto de vista de su contenido por la autora del presente estudio. $^{9}$

2. Escala Psicosocial del cuidador. Esta es una escala likert que mide dos tipos de variables; las socioeconómicas, entre las que se encuentran las siguientes: recursos de apoyo, tiempo libre, recursos económicos, satisfacción de necesidades, conflictos familiares, relaciones sociales y dificultades laborales; y las variables psicológicas, donde se encuentra: la angustia, irritabilidad, vergüenza, culpabilidad, miedo, soledad, resentimiento y desesperanza.

Los datos recogidos fueron procesados de forma computarizada y los resultados se expresaron en porcentajes. A aquellos datos que resultaron apropiados se les aplicó la prueba ji cuadrado de independencia, calculándose el valor $\mathrm{p}$ de la probabilidad asociada con un nivel de significación á=0,05.

\section{RESULTADOS}

La mayor parte de los cuidadores (55,7 \%) tenía entre los 40 y 59 años, seguido del grupo de 60 años y más donde estaba el 36, $0 \%$; sólo eran jóvenes, de menos de 40 años el 8,3\%. En relación con el sexo, 70,5\% eran mujeres y 29,5\%, hombres. La mayoría de los cuidadores $(55,7 \%)$ estaban casados, solteros el 41,0 $\%$ y viudos el $3,3 \%$.

El nivel escolar predominante fue el universitario $(49,2 \%)$, seguido de los de nivel educacional medio $(24,6 \%)$ y de los de nivel preuniversitario (18\%). Sólo el 8,2 $\%$ tenía un nivel de enseñanza primario.

En relación con el vínculo laboral, 47,5 \% de los cuidadores se encontraba trabajando, $34,4 \%$ se había jubilado y $18,0 \%$ eran amas de casa.

El $68,9 \%$ cuidaba sólo a una persona enferma mientras $24,6 \%$ cuidaba a dos e incluso cuatro personas de la muestra $(6,5 \%)$ se encontraban cuidando a más de 2 enfermos..

En cuanto a las motivaciones que señalaron los cuidadores para hacer su tarea, se encontraba en primer lugar los lazos afectivos (73,8 \%), después, los que lo hacían por estar cumpliendo un deber moral ( $24,6 \%$ ) y sólo un pequeño porcentaje $(1,6$ $\%$ ) perseguía algún interés material con el cuidado del enfermo.

Con respecto a si este grupo poseía información acerca de la enfermedad y su manejo, se apreció que en $67,2 \%$ de los casos era nula, 31,2 \% tenía alguna información pero insuficiente y sólo 1,6 \% consideraba que la información que tenía era suficiente.

Los problemas de salud predominantes que en los últimos 6 meses fueron los problemas nerviosos $(31,1 \%)$ seguidos de los problemas óseos y musculares (23, $.0 \%$ ) y las cefaleas $(19,6 \%)$. Además se observó que $18,0 \%$ tenía problemas cardíacos y el 8,2 \% había padaecido de hipertensión arterial. 
La gran mayoría de los casos, 77,0 \% no tenía experiencia en el cuidado de una persona enferma y $23,0 \%$ lo había hecho antes.

La estrategia de afrontamiento más utilizaba fue la búsqueda de ayuda por parte de familiares $(42,5 \%)$, seguida de la búsqueda de ayuda de otras personas (29,5\%) y por último, la búsqueda de información en el 28,0 \% .

En relación con el tiempo de cuidador , 36,1 \% llevaba menos de 1 año cuidando al enfermo, $32,8 \%$ de 1 a 4 años y 31,1\% 5 o más años.

Se encontró que el 73,8 \% de los cuidadores eran los hijos de los enfermos, cónyuges en el 13,2 \% y por último los nietos y los hermanos, 6,5 \% cada uno.

Entre los resultados de las afectaciones de índole socioeconómica, se observó que más del $50 \%$ no disponía de tiempo libre y en orden deecreciente de porcentaje, los problemas económicos y los conflictos familiares (tabla 1 ).

Con respecto a la afectación psicológica, la angustia, el miedo y la desesperanza fueron los sentimientos negativos que más experimentaban los cuidadores, aunque también la mayoría sentían ira y sentimiento de soledad (tabla 2).

\section{DISCUSIÓN}

En la mayoría de los estudios realizados y en el que aquí se describe, la edad promedio predominante de los cuidadores estuvo en el rango de 40 a 59 años. Así en uno de los estudios de mayor número de observaciones que se ha efectuado, en 2089 cuidadores informales, la media de edad fue de 57 años. ${ }^{10}$ Otros dos estudios demostraron que la edad promedio de los cuidadores familiares de ancianos con demencia tipo Alzheimer era de 50 y de 59 años respectivamente. Estos resultados apuntan hacia la necesidad de velar por la salud de estos cuidadores que se encuentran en una edad que se caracteriza por la aparición de enfermedades crónicas las cuales están asociadas al estrés y en este caso la carga que genera el cuidado y la vulnerabilidad que presentan pudiera llevarlos a padecer afecciones físicas y psicológicas. A continuación estuvo el grupo de 60 años y más para un 33 $\%$, lo que indica la presencia de cuidadores que también son adultos mayores con la vulnerabilidad física y psicológica que de por sí tienen las personas de este grupo de edad, lo que puede hacerlos más propensos a presentar alteraciones como consecuencia del cuidado del enfermo.

El sexo femenino es el predominante en los cuidadores y esto se ha encontrado en múltiples estudios así como el hecho de que son las hijas las encargadas mayormente del cuidado de los ancianos dependientes. En un estudio realizado en 70 cuidadores familiares de enfermos de Alzheimer, 84,3\% eran del sexo femenino y el $64 \%$ eran hijos. ${ }^{3}$ Otro estudio registró el

$80 \%$ de cuidadoras mujeres donde el $48 \%$ eran hijas. ${ }^{8}$ Estos resultados están determinados fundamentalmente, por factores culturales que le han asignado a la mujer el papel de cuidar, ya que desde edades tempranas es entrenada para el cuidado de los hijos y porque a ellas se les considera más "preparadas" para asumir esta tarea. Sin embargo la incorporación de la mujer a la vida social, entre otros factores ha llevado aparejada la presencia en aumento de los cuidadores hombres. 
Otra característica de los cuidadores informales, es que son personas casadas. Esto pudiera deberse a que ya tienen edad suficiente para haber formado su propia familia con toda la responsabilidad que esto entraña. Tal situación multiplica sus responsabilidades familiares y pudiera llevar a una mayor carga.

El presente estudio arrojó un alto porcentaje de cuidadores de nivel escolar superior lo que no se ha confirmado en estudios internacionales donde se encuentra que el $56 \%$ tiene nivel primario y sólo el $16 \%$ había terminado estudios universitarios. ${ }^{11}$ Otro estudio también revela que el $80 \%$ de los cuidadores tiene nivel primario de educación. Esta diferencia pudiera estar dada por el alto índice educacional que existe hoy en Cuba, siendo esto un factor protector para la salud de los cuidadores debido a que tienen más recursos cognitivos para enfrentar el cuidado y mayor acceso a la información necesaria para asumir esta labor.

Una característica que sí coincide con otros estudios nacionales e internacionales es que alrededor de la mitad de los cuidadores mantienen vínculo laboral. ${ }^{8}$ Muchos no continúan laborando y a veces se jubilan antes de lo previsto por tener que asumir esta tarea, pero otros siguen trabajando por razones económicas y/o profesionales. Esta situación amplía la diversidad de tareas y el esfuerzo que deben realizar, lo que requiere de una buena planificación del tiempo. Otro estudio muestra que cerca de la mitad de los cuidadores seguían trabajando, el $9 \%$ había dejado de hacerlo por la necesidad de cuidar al anciano y el $20 \%$ había tenido que alterar sus esquemas laborales. ${ }^{6}$

Una particularidad encontrada en el estudio fue la cantidad de personas receptoras de cuidado. La mayoría de los cuidadores atendía sólo a un enfermo, sin embargo el 30 \% cuidaba a dos o más personas lo que pudiera incrementar lógicamente la carga de los cuidadores debido a que se multiplican las tareas y el esfuerzo físico y mental que requieren.

Con respecto a la motivación para cuidar al anciano, son diversas las razones para hacerlo, pero se ha visto que la mayoría están impulsados por relaciones de afecto con la persona cuidada. En un estudio $58 \%$ de lo cuidadores alegan que el enfermo que cuidaban era un "ser que necesitaba ayuda" y sólo 15 \% "porque era su obligación". ${ }^{3}$ No existen muchos estudios que profundicen en las motivaciones reales del cuidador así como en el tipo de relación cuidador-familiar, sin embargo es un aspecto que pudiera incidir en la calidad del cuidado ofrecido y en la carga del cuidador.

Entre las variables que más se estudian actualmente así como su repercusión en la carga, están las estrategias de afrontamiento. Los cuidadores participantes en este estudio utilizaron estrategias de búsqueda de apoyo externo solicitando la ayuda de otras personas, tanto familiares como no familiares y un número de cuidadores buscaba información acerca de la enfermedad de su familiar. Se observó que los cuidadores no utilizaban estrategias de apoyo interno para afrontar el cuidado. Un estudio plantea que los cuidadores utilizan una combinación de afrontamiento centrado en la emoción y en el problema en lo referente al comportamiento del paciente. La respuesta más común es "desear que la situación no existiera" e "intentar ver las cosas de otra manera." 12 Se ha argumentado con respecto a esta variable que los cuidadores varones se distancian más emocionalmente y buscan ayuda entre otros miembros de la familia y la mayor utilización por parte de las mujeres de estrategias de afrontamiento de tipo evitativo. ${ }^{13}$ Se ha demostrado que una de las variables que incide en la carga del cuidador es "la capacidad personal para enfrentarse a la adversidad". ${ }^{14}$ 
Otros investigadores describen diferentes tipos de estrategias de afrontamiento: aislamiento del problema, conformidad, disconformidad/incomprensión, estrategias de entrenamiento cognitivo, evasión, y reestructuración cognitiva. ${ }^{15}$ La diversidad de estudios relacionados con las estrategias utilizadas por los cuidadores ha demostrado que varían de acuerdo con la edad, el sexo, el tiempo de cuidados, el vínculo laboral, el nivel educacional y otras variables del cuidador, y es un aspecto importantísimo en la estimación de la carga. Las mujeres más jóvenes, trabajadoras, con un mayor nivel de estudios, enfrentan mejor el cuidado de su familiar.

Con respecto al tiempo de cuidado, los cuidadores en su mayoría llevaban menos de un año en esta labor. Esta es una variable que se ha medido en diferentes estudios y se ha visto su influencia en el grado de aceptación y adaptación a la enfermedad de la persona cuidada que tiende a aumentar, a medida que transcurre el tiempo. ${ }^{16}$

Otras dos variables estudiadas fueron la experiencia en cuidar a un enfermo crónico y el conocimiento acerca de la enfermedad, ambas son herramientas importantes para la garantía de una mayor calidad del cuidado. En el presente estudio, los cuidadores no contaban con los conocimientos ni habían desarrollado las habilidades necesarias para el cuidado óptimo de su familiar.

Ha sido ampliamente investigada la percepción de la salud de los cuidadores ya que se ha afirmado que la salud del cuidador se afecta tanto en el orden físico como psicológico. Los problemas de salud referidos por los cuidadores del presente estudio corrobora lo planteado acerca de que las quejas somáticas son múltiples, entre ellas, el dolor crónico del aparato locomotor, la cefalea tensional, la astenia, la fatiga crónica, la alteración del ciclo sueño-vigilia, el insomnio y otros. Además, algunos estudios demuestran el deterioro de la función inmune y la mayor predisposición a otro tipo de enfermedad como la ulcerosa y la cardiovascular. A su vez, se ha indicado que los cuidadores tienen una alta tasa de automedicación, especialmente de diversos tipos de psicofármacos y analgésicos. ${ }^{17}$ Los resultados de una investigación indican que el efecto mayor del cuidado de un familiar es en la salud psicológica que afecta el $67,0 \%$ de los cuidadores y un número similar, 65,0 $\%$ comunica problemas con su salud física. ${ }^{18}$ Los cónyuges de enfermos de Alzheimer muestran una alta prevalencia de depresión, 25,0 \%, que asciende hasta $32,0 \%$ durante un seguimiento de 5 años realizado por Kiecolt-Glaser y colaboradores. Estos autores dicen que el grado de sobrecarga se correlaciona significativamente con la morbilidad psicológica en los cuidadores de enfermos de Alzheimer. ${ }^{19}$

La repercusión psicológica del cuidado de una persona mayor dependiente no se limita a la depresión y a la ansiedad, que han sido las alteraciones más estudiadas; también se ha encontrado que experimentan sentimientos negativos que les resulta difícil manejar. Así por ejemplo, un estudio encuentra en $42,0 \%$ de los cuidadores, la aparición de tristeza y deseos de llorar, 32,0 \% confiesa tener sentimientos de culpabilidad si no se encontraban al lado del paciente. Las reacciones emocionales adversas encontradas fueron: ansiedad $(58,0 \%)$, depresión $(50,0 \%)$, miedo $(35,0$ $\%)$, frustración $(32,0 \%)$, resentimiento $(29,0 \%)$, impaciencia $(25,0 \%)$ y culpabilidad $(10,0 \%)$, las cuales se encontraban sin diagnosticar y sin tratar. ${ }^{20}$ Otro estudio revela que $26,7 \%$ de los cuidadores se sentían solos en el cuidado, $60,0 \%$ sentía miedo por el futuro, y 20,7 \% tenía depresión.

Es necesario incorporar a los programas de intervención psicológica con cuidadores, la forma de identificar y manejar de una manera adecuada estos sentimientos, que 
interfieren en el cuidado y dañan la calidad de vida, tanto del cuidador como del receptor de cuidados.

Existen otras variables que se enmarcan en lo socioeconómico que también se afectan con el cuidado de una persona dependiente, se trata de los ingresos monetarios, la vida social, laboral y familiar y el empleo del tiempo libre. Un estudio plantea que las principales consecuencias de la atención a familiares mayores dependientes eran la restricción en el tiempo libre y en actividades sociales, las cargas económicas y las dificultades laborales, entre otras. ${ }^{21}$

En el presente estudio se encontró que una de las mayores afectaciones derivadas del cuidado del adulto mayor dependiente, eran los conflictos familiares, lo cual coincide con los resultados de otras inverstigaciones realizadas y superado sólo por la poco disponibilidad de tiempo libre. ${ }^{22} \mathrm{Si}$ bien la familia es la principal proveedora de cuidados y tanto sus miembros como el propio anciano desea permanecer en ella y obtener de esta toda la atención, cuando llega el momento en que pierde su autonomía y su capacidad para valerse, no dejan de suscitarse contradicciones y en algunos casos de carácter severo, debido a una serie de cambios que debe asumir la familia para lo cual pocas veces está preparada. Pueden aparecer conflictos familiares por el desacuerdo entre la persona que cuida y otros familiares, por el comportamiento, por las decisiones y actitudes de unos y otros hacia la persona mayor o por la forma en que se proporcionan los cuidados. Además, ocurre la "inversión de roles", produciéndose un cambio en la dirección en la que se produce el cuidado habitual de padres e hijos, lo cual requiere de un cambio de mentalidad respecto al tipo de relación padres-hijos y exige del cuidador un esfuerzo de adaptación. El cuidado de la persona mayor dependiente implica muchas tareas, tiempo y dedicación, que en ocasiones la pareja o los hijos del cuidador le reclaman a este. Otro elemento que incide en la aparición de este problema es la percepción del cuidador de que no recibe apoyo suficiente tanto en recursos, en ayuda, y desde el punto de vista moral, por parte del resto de la familia. La satisfacción de las necesidades personales es otro aspecto que se ve afectado, el cuidador poco a poco va centrando su vida en el cuidado del enfermo y se va olvidando de sí mismo. Así en un estudio se encuentra que el 36,0 \% de cuidadores tenían afectada su intimidad. Otra investigación informa que para cuidar mejor al enfermo, el cuidador deja de lado sus propias necesidades y argumenta que el refuerzo de los cuidadores informales de pacientes con demencia proviene del propio acto de cuidar, más que de la mejoría del paciente. ${ }^{23}$

Finalmente se puede concluir que la mayor parte de las características del perfil de los cuidadores del presente estudio coinciden con las encontradas en otros trabajos como son el sexo femenino, tener entre 40 y 59 años, ser hija del enfermo, etado civil casado y sin vínculo laboral. Se conoció que son razones afectivas las que impulsan a ofrecer los cuidados a pesar de no contar con una experiencia previa en este rol y de presentar afectaciones múltiples relacionadas con la salud física y mental así como en el orden social y económico, por lo que se hace inminente la búsqueda de alternativas de apoyo a estos cuidadores que no cuentan con un nivel mínimo de información acerca de la demencia y de los problemas que presenta el adulto mayor al que ofrecen sus cuidados, a pesar de tener un nivel de enseñanza superior en su mayoría.

\section{REFERENCI AS BI BLI OGRÁFICAS}

1- Alfonso Fraga JC. El descenso de la fecundidad en Cuba: de la primera a la segunda transición demográfica. Rev Cubana Salud Pública. 2006; 32 (1):2-6. 
2- Llibre JJ. Estrategias de investigación en la enfermedad de Alzheimer. Rev Cubana Med Gen Integr. 2002; 18(4):13-20.

3- Vidal Gutiérrez D, Zaval Gutiérrez M, Castro Salas M, Quiroga López $P$, Klaasen Pinto G. El significado del paciente con demencia para el cuidador en una comunidad urbana y rural. Rev Servicio Social. 1999; 1(2): 1-10.

4- Guzmán J M, Huenchuan S. Políticas hacia las familias con adultos mayores: el desafío del derecho al cuidado en la edad avanzada. Reunión de expertos. Santiago de Chile: CELADE- CEPAL;2005.

5- De los Reyes MC. Construyendo el concepto de cuidador de ancianos. Foro de Investigación, Envejecimiento de la población. Curitiba, Brasil: MERCOSUR; 2001.

6- Bermejo F, Rivera J, Pérez del Molino F. Aspectos familiares y sociales en la demencia. Med Clín (Barc). 1997; 109: 140-6.

7- Izal-Fernández M, Montorio-Cerrato I. Evaluación del medio y del cuidador del demente. En: Peña TJ, editor. Del Ser. Evaluación neuropsicológica y funcional de la demencia. Barcelona: Proas; 1994. p 201-22.

8- Artaso Irigoyen B, Goñi Sarriés A, Gómez Martínez AR. Sobrecarga del cuidador informal del paciente con demencia: demanda en un Centro de Día Psicogeriátrico en Navarra. Geriatrika. 2001; 17 (2):69-73,39-43.

9- Espín Andrade AM, Seco Mata T. Metodología de intervención educativoterapéutica en cuidadores de ancianos con demencia de Alzheimer. Rev Arg Geriatría. 1998; 18: 18-27.

10- Bermejo FP, Rivera J N, Trincado RS, Olazarán J, Morales JM. Aspectos sociales y familiares del paciente con demencia. Datos de un estudio poblacional en dos zonas de Madrid. Madrid: Díaz de Santos; 1997.

11- Carod-Artal FJ, Egido- Navarro J A, González- Gutiérrez JL, Varela de Seijas E. Percepción de la sobrecarga a largo plazo en cuidadores de supervivientes de un ictus. Rev Neurol. 1999; 28 (12): 1130-8.

12- Samele C, Manning N. Nivel de carga del cuidador entre los familiares del enfermo mental en el sur de Verona. Eur Psychiatry. 2000; 15: 196-204.

13- Harwood DG, Barrer WW, Ownbby RL, Bravo M, Aguero H, Duara R. Predictors of positive and negative appraisal among Cuban American caregivers of Alzheimer's disease patients. Int J Geriat Psychiatry. 2000; 15:481-7.

14- Dunkin J, Anderson-Hanley C. Dementia caregiver burden. A review of the literature and guidelines for assessment and intervention. Neurology. 1998;51( suppl 1):53-60.

15- Deví J, Ruíz I. Modelos de estrés y afrontamiento en el cuidador del enfermo con demencia. Rev Multidisciplinar Gerontol. 2002;12(1): 10-5.

16- Bengozar Torres MC, Serra Desfilis E. Empleo de estrategias de afrontamiento en cuidadores familiares de ancianos con demencia. Rev Esp Geriat Gerontol. 1997; 32(5): 257-69. 
17- Schulz R, Beach SR. Caregiving as a risk factor for mortality: The Caregiver Health Effects Study. JAMA. 1999;282(23):2215-9.

18- Loukissa DA. Family burden in chonic mental illness: a review of research studies. J Adv Nurs. 1995;21:248-55.

19- Medalie JH. The patient and family adjustment to chronic disease in the home. Disabil Reabil. 1997; 19:163-70.

20- Anderson CS, Linto J, Stewart-Wynne EG. A population- based assessment of the impact and burden of caregiving for long-term stroke survivors. Stroke. 1995; 26: 843-9.

21- Montorio I, Díaz P, Fernández MI. Programas y servicios de apoyo a familiares cuidadores de ancianos dependientes. Rev Esp Geriat Geron. 1995; 30(3): 157-68.

22- Parks SM, Novielli KD. A practical guide to caring for caregivers. Am Fam Physician. 2000;62:2613-20.

23- Ginsberg J, Martínez MF, Mendoza A, Pabón J L. Carga subjetiva percibida por el cuidador y su relación con el nivel de deterioro de pacientes con diagnóstico de demencia. Influencia de edad, estilo de personalidad y tipo de cuidador. Archivos Venezolanos Psiquiatría Neurología. 2005; 51(104).

Recibido: 24 de enero de 2007.

Aprobado: 21 de marzo de 2008.

Ana Margarita Espín Andrade. Escuela Latinoamericana de Medicina. Calle 52 No. 4120 e/ 41y 43. Playa. La Habana, Cuba.Telf.: 209-5966,

E-mail: amespin@infomed.sld.cu, anam@elacm.sld.cu

Tabla 1. Resultados de la afectación de variables socioeconómicas

\begin{tabular}{|l|l|l|}
\hline Variables socioeconómicas & No. de casos & $\%$ \\
\hline Apoyo insuficiente & 39 & 59 \\
\hline Poco tiempo libre & 54 & 82 \\
\hline Problemas económicos & 42 & 64 \\
\hline Insatisfacción de necesidades & 39 & 59 \\
\hline Conflictos farriliares & 40 & 60 \\
\hline Pocas relaciones sociales & 38 & 57 \\
\hline Dificultades laborales & 36 & 54 \\
\hline
\end{tabular}


Tabla 2. Resultado de la afectación de variables psicológicas

\begin{tabular}{|l|l|l|}
\hline Variables psicológic as & No. de casos & $\%$ \\
\hline Angustia & 54 & 82 \\
\hline Ira & 42 & 64 \\
\hline Vergïenza & 9 & 13 \\
\hline Culpabilidad & 17 & 26 \\
\hline Miedo & 50 & 75 \\
\hline Soledad & 40 & 60 \\
\hline Resentimiento & 15 & 23 \\
\hline Desesperanza & 44 & 67 \\
\hline
\end{tabular}

